\title{
Step Training Reinforces Specific Spinal Locomotor Circuitry in Adult Spinal Rats
}

\author{
Ronaldo M. Ichiyama, ${ }^{1,3}$ Grégoire Courtine, ${ }^{2,3}$ Yury P. Gerasimenko, ${ }^{3,6}$ Grace J. Yang, ${ }^{3}$ Rubia van den Brand, ${ }^{2,3}$ \\ Igor A. Lavrov, ${ }^{3}$ Hui Zhong, ${ }^{3}$ Roland R. Roy, ${ }^{3,4}$ and V. Reggie Edgerton ${ }^{3,4,5}$ \\ ${ }^{1}$ Institute of Membrane and Systems Biology, University of Leeds, Leeds LS2 9JT, United Kingdom, ${ }^{2}$ Experimental Neurorehabilitation Laboratory, \\ University of Zurich, CH-8032 Zurich, Switzerland, Departments of ${ }^{3}$ Physiological Science and ${ }^{4}$ Neurobiology and ${ }^{5}$ Brain Research Institute, University of \\ California, Los Angeles, Los Angeles, California 90095, and 'Pavlov Institute of Physiology, Russian Academy of Sciences, St. Petersburg 199034, Russia
}

Locomotor training improves function after a spinal cord injury both in experimental and clinical settings. The activity-dependent mechanisms underlying such improvement, however, are sparsely understood. Adult rats received a complete spinal cord transection (T9), and epidural stimulation (ES) electrodes were secured to the dura matter at L2. EMG electrodes were implanted bilaterally in selected muscles. Using a servo-controlled body weight support system for bipedal stepping, five rats were trained $7 \mathrm{~d} /$ week for 6 weeks $(30 \mathrm{~min} / \mathrm{d})$ under quipazine $(0.3 \mathrm{mg} / \mathrm{kg})$ and ES $(\mathrm{L} 2 ; 40 \mathrm{~Hz})$. Nontrained rats were handled as trained rats but did not receive quipazine or ES. At the end of the experiment, a subset of rats was used for c-fos immunohistochemistry. Three trained and three nontrained rats stepped for $1 \mathrm{~h}$ (ES; no quipazine) and were returned to their cages for $1 \mathrm{~h}$ before intracardiac perfusion. All rats could step with ES and quipazine administration. The trained rats had higher and longer steps, narrower base of support at stance, and lower variability in EMG parameters than nontrained rats, and these properties approached that of noninjured controls. After $1 \mathrm{~h}$ of stepping, the number of FOS + neurons was significantly lower in trained than nontrained rats throughout the extent of the lumbosacral segments. These results suggest that training reinforces the efficacy of specific sensorimotor pathways, resulting in a more selective and stable network of neurons that controls locomotion.

Key words: epidural electrical stimulation; locomotion; spinal cord; rats; training; c-fos

\section{Introduction}

Loss of motor function below the level of a spinal cord injury (SCI) is one the most devastating effects of such an injury. Locomotor training has been used successfully to promote functional recovery in both laboratory and clinical settings (for review, see Edgerton et al., 2006). Nevertheless, the mechanisms underlying this activity-dependent plasticity in the lumbosacral spinal cord are only sparsely understood, especially in adult models of SCI. Because of technical challenges, the study of the central neural circuitry and mechanisms involved in spinal locomotion has been generally confined to acute experiments (i.e., in vitro neonatal and decerebrate preparations) (Kiehn, 2006).

We previously showed that epidural electrical stimulation (ES) at spinal cord segment L2 can facilitate coordinated, weightbearing stepping after a complete midthoracic spinal cord transection in adult rats (Ichiyama et al., 2005). We also showed that ES in combination with the administration (intraperitoneal) of the 5-HT agonist quipazine produces a greater number of coor-

Received Feb. 27, 2008; revised May 30, 2008; accepted June 2, 2008.

This work was supported by the State of California Roman Reed Funds, Christopher and Dana Reeve Foundation Grant VEC-2005, Russian Foundation for Basic Research (RFBR) Grant N 07-04-00526, and RFBR-US Civilian Research \& Development Foundation Grant N 07-04-91106.

Correspondence should be addressed to Dr. Ronaldo M. Ichiyama, Institute of Membrane and Systems Biology, University of Leeds, Leeds LS2 9JT, UK. E-mail: r.m.ichiyama@leeds.ac.uk.

DOI:10.1523/JNEUROSCI.1881-08.2008

Copyright $\odot 2008$ Society for Neuroscience $\quad$ 0270-6474/08/287370-06\$15.00/0 dinated plantar steps on a moving treadmill when compared with ES alone or quipazine alone (Gerasimenko et al., 2007; Ichiyama et al., 2008). In those studies, ES plus quipazine acutely changed the physiological state of the lumbosacral spinal cord in a manner that enabled coordinated locomotion to occur in response to proprioceptive input without input from the brain. Combining ES and quipazine administration thus provides a novel opportunity for studying the circuitry of spinally controlled locomotion in an in vivo, awake, adult mammalian preparation.

In the present investigation, we used ES in combination with quipazine and daily bipedal step training to identify and characterize alterations in behavioral, physiological, and morphological parameters associated with activity-dependent plasticity in the spinal cord of the adult rat. We demonstrate that one of the mechanisms that underlies the step-training induced reorganization of the functional properties in the adult mammalian spinal cord after elimination of input from the brain is a more selective reinforcement of a subset of neurons activated during locomotion. Trained spinal rats developed a unique strategy to produce successful steps compared with nontrained spinal animals. A systematic reduction in c-fos immunoreactivity throughout the extent of the lumbosacral segments in trained versus nontrained spinal rats accompanied the improved locomotion observed in the trained rats.

\section{Materials and Methods}

Animals and animal care. All experimental procedures were approved by the University of California Los Angeles Chancellor's Animal Research 
Committee and complied with the guidelines of the National Institutes of Health Guide for the Care and Use of Laboratory Animals. Adult female Sprague Dawley rats (200-250 g) were assigned randomly to a control noninjured ("noninjured"; $N=10)$ or a spinal cord transected $(N=10)$ group. Five rats in the spinal group were step trained ("trained"), and five were nontrained ("nontrained"). All rats were housed individually on a $12 \mathrm{~h}$ light/dark cycle, with access to food and water ad libitum.

Surgical procedures and postsurgical care. Procedures have been described in detail previously (Ichiyama et al., 2005). Briefly, rats were anesthetized deeply with a combination of ketamine $(100 \mathrm{mg} / \mathrm{kg})$ and xylazine $(10 \mathrm{mg} / \mathrm{kg})$, and maintained at this level with supplemental doses of ketamine (intraperitoneal). Under aseptic conditions, a partial laminectomy was performed at midthoracic level ( $\sim \mathrm{T} 7-\mathrm{T} 9)$, and the spinal cord was completely transected. A partial laminectomy was performed over spinal segment L2. One Teflon-coated stainless-steel wire (AS632; Cooner Wire) was passed under the spinous processes and above the dura matter of the remaining vertebrae between the partial laminectomy sites. A small portion ( $\sim 1 \mathrm{~mm}$ notch) of the Teflon coating was removed, and the created electrode was secured at the midline of the spinal cord by suturing the wire to the dura matter above and below L2.

Bipolar intramuscular EMG electrodes using the same wire type as above were inserted in the deep midbelly of the vastus lateralis (VL), semitendinosus (St), medial gastrocnemius (MG), and tibialis anterior (TA) muscles bilaterally as described previously (Hodgson et al., 2005). All electrode wires were connected to an amphenol connector cemented to the skull of the animal. Rats recovered in a warm incubator after surgery. After recovery, rats were kept in individual cages, their bladder expressed three times per day for the first 2 weeks and two times daily thereafter.

Locomotor training. Each trained rat received an intraperitoneal injection of quipazine $(0.3 \mathrm{mg} / \mathrm{kg}) 10-15 \mathrm{~min}$ before each training session as established previously (Ichiyama et al., 2008). Continuous ES was delivered at $40 \mathrm{~Hz}$ with $200 \mu$ s duration rectangular pulses, as previously determined (Ichiyama et al., 2005). An upper body harness was used to position the rats over a treadmill belt and partially support their body weight during bipedal locomotion. An automated, servo-controlled body weight support system provided the optimal amount of body weight support that each rat needed to step (Timoszyk et al., 2005). Rats were trained $7 \mathrm{~d} /$ week, $30 \mathrm{~min} / \mathrm{session}$ for 6 weeks, starting $7 \mathrm{~d}$ after transection and implantation. Treadmill belt speed was increased progressively from 6 to $21 \mathrm{~cm} / \mathrm{s}$. By week 3 , all trained rats were able to step at $21 \mathrm{~cm} / \mathrm{s}$ for $30 \mathrm{~min}$. Nontrained rats were housed similarly to the trained rats but did not receive daily quipazine or ES treatment and were not placed in the treadmill apparatus, except for the final testing session when all rats were tested under the same conditions.

Behavioral testing and analysis. The amphenol headplug was connected to a Grass S88 Stimulator (Grass Instruments) through a stimulus isolation unit (Grass SIU5; Grass Instruments). Muscle twitch threshold and the threshold for inducing locomotor activity under high-frequency (40 $\mathrm{Hz})$ ES were determined. EMG signals $(2 \mathrm{kHz})$ were amplified, filtered (10-1000 $\mathrm{Hz}$ bandpass), and used to compute the duration and mean amplitude of identified bursts (Courtine et al., 2008).

All kinematic data were collected in one session at the end of the 6 week training period. Three-dimensional video recordings $(100 \mathrm{~Hz})$ were made using four cameras (Basler Vision Technologies) oriented at 45 and $135^{\circ}$ bilaterally with respect to the direction of locomotion. Reflective markers were attached bilaterally at the spine, iliac crest, greater trochanter, lateral condyle, lateral malleolus, the distal end of the fifth metatarsal, and the lateral surface of the fifth metatarsal. SIMI motion capture software (SIMI Reality Motion Systems) was used to obtain threedimensional coordinates of the markers. The body was modeled as an interconnected chain of rigid segments, and joint angles generated accordingly. All rats were tested once with no stimulation and no quipazine, and with quipazine $(0.3 \mathrm{mg} / \mathrm{kg}$, i.p.) plus stimulation $(\mathrm{L} 2 ; 40 \mathrm{~Hz})$. Rats were stepped bipedally on a treadmill at speeds from 8 to $25 \mathrm{~cm} / \mathrm{s}$. Ten consistent step cycles were extracted from a continuous sequence of stepping for each animal. Joint angular and limb linear displacements were computed for those steps together with established gait parameters (Courtine et al., 2005). c-fos immunohistochemistry. FOS immunoreactivity of spinal neurons was determined in three trained and three nontrained rats. After a $60 \mathrm{~min}$ bout of continuous hindlimb bipedal stepping (ES; no quipazine), rats were returned to their cages and $\sim 60$ min later were anesthetized and intracardially perfused with $4 \%$ paraformaldehyde in phosphate buffer. The procedures of FOS immunohistochemistry (IHC) have been described in detail previously (Ahn et al., 2006). Briefly, after perfusion, spinal cords were grossly dissected with the vertebral column and postfixed overnight $\left(4^{\circ} \mathrm{C}\right)$. Spinal cords then were dissected carefully, and cryoprotected in $30 \%$ sucrose in PBS for at least $3 \mathrm{~d}$. Segments T12 through S1 were mounted and frozen, and $25 \mu \mathrm{m}$ coronal sections were obtained using a cryostat. Triplicate sections of each spinal segment for a trained and a nontrained rat were processed simultaneously for FOS immunoreactivity. Free-floating sections were submerged in $0.3 \% \mathrm{H}_{2} \mathrm{O}_{2}$ (10 min) and subsequently washed in PBS (three times for $10 \mathrm{~min}$ ). Sections were incubated in the primary antibody to c-fos protein product FOS (rabbit, polyclonal; Santa Cruz Biotechnology) at 1:200 overnight at room temperature. Sections were then washed in PBS (30 min) and incubated in the secondary antibody (biotinylated donkey anti-rabbit; Jackson ImmunoResearch) at 1:100 for $1 \mathrm{~h}$. After PBS washes (30 min), sections were incubated for $1 \mathrm{~h}$ in a 1:100 Vectastain Elite ABC (avidinbiotin peroxidase complex) solution (Vector Laboratories). Sections again were washed in PBS and reacted with DAB (diaminobenzidine) solution (Sigma-Aldrich) for $\sim 2-3 \mathrm{~min}$. Finally, sections were rinsed in PBS (30 min), mounted on microscope slides, air-dried, and coverslipped with Permount. FOS + nuclei were counted manually using camera lucida techniques and superimposed onto Molander's cytoarchitectonic maps of the rat thoracic and lumbosacral cord (Molander et al., 1984). Counts were performed for all three sections for each spinal segment for each animal, and the average was used for statistical analysis.

Statistical analyses. Differences in EMG and kinematic parameters between trained and nontrained groups and body sides were determined using repeated measures ANOVAs with groups as a factor and body side as the repeated measure. A two-way factorial ANOVA was performed to determine differences in number of FOS + nuclei for spinal segments $(2 \times 9)$ and laminas $(2 \times 12)$ between trained and nontrained rats.

\section{Results}

Step training under ES and quipazine resulted in a unique pattern of locomotion (Fig. 1). The steps in the trained rats were higher (Fig. 1C) $(p<0.01)$ and longer (Fig. 1D) $(p<0.05)$ than in nontrained rats. In addition, the total quantity of hindlimb joint movement per gait cycle [i.e., the algebraic sum of changes in joint angle amplitude at the hip, knee, ankle, and metatarsophalangeal joint (MTP)] was greater in trained than nontrained rats (Fig. 1E). Trained rats developed a gait pattern with a narrower stance width than nontrained rats (Fig. $2 E$ ) (i.e., the foot was placed along a line that coincided with the body midline). The gait timing variability, as assessed by the coefficient of variability of cycle duration (Fig. $1 B$ ) and stance duration variability (Fig. $2 D)$ was similar in no-injury and spinal trained rats, but was twofold higher in nontrained rats $(p<0.01)$. Likewise, trained and no-injury rats showed out-of-phase coordination between left and right limb oscillations (Fig. $2 B)(p<0.05)$ and between the left and right flexor (St) bursts (Fig. $2 C)(p<0.01)$, whereas left-right coordination was significantly altered in nontrained rats. Consistent with the observed facilitation of flexion during the swing phase of gait (Fig. $1 A$ ), the TA EMG amplitude was higher $(p<0.001)$ in the trained than nontrained rats (Fig. $1 F)$.

FOS + nuclei were present in all laminas of segments T12 to S1 of both spinal trained and nontrained rats (Fig. $3 A, B$ ). The number of FOS + nuclei was not significantly different between trained and nontrained rats for spinal segments T12 and T13 (Fig. $3 C)$. There was, however, an overall greater number of FOS+ nuclei in nontrained than trained rats in all segments caudal to T13 $(p<0.001)$. There were no significant differences in the 
A NON-INJURED

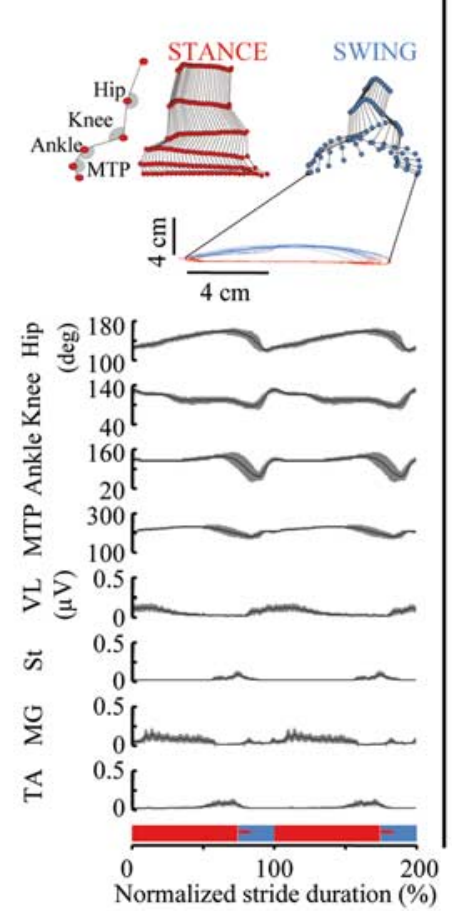

\section{COMPLETE SPINAL CORD TRANSECTION}

TRAINED

(no Q, no ES)
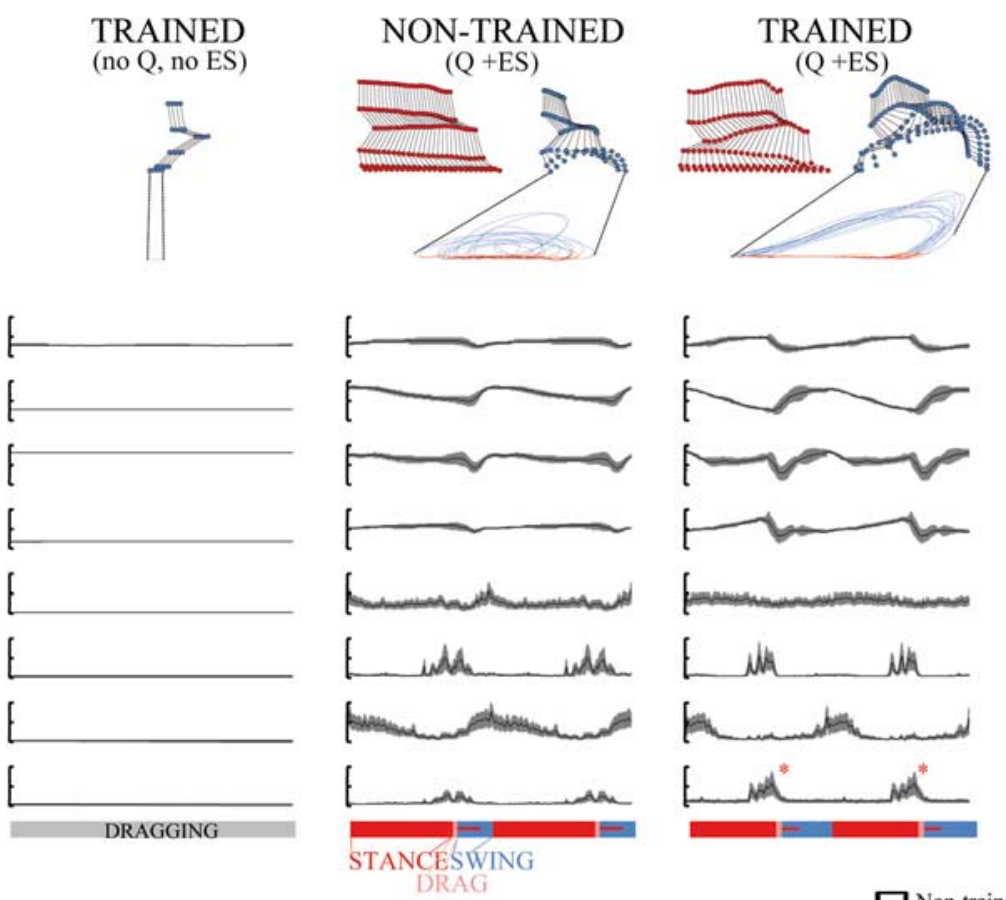

DRAG
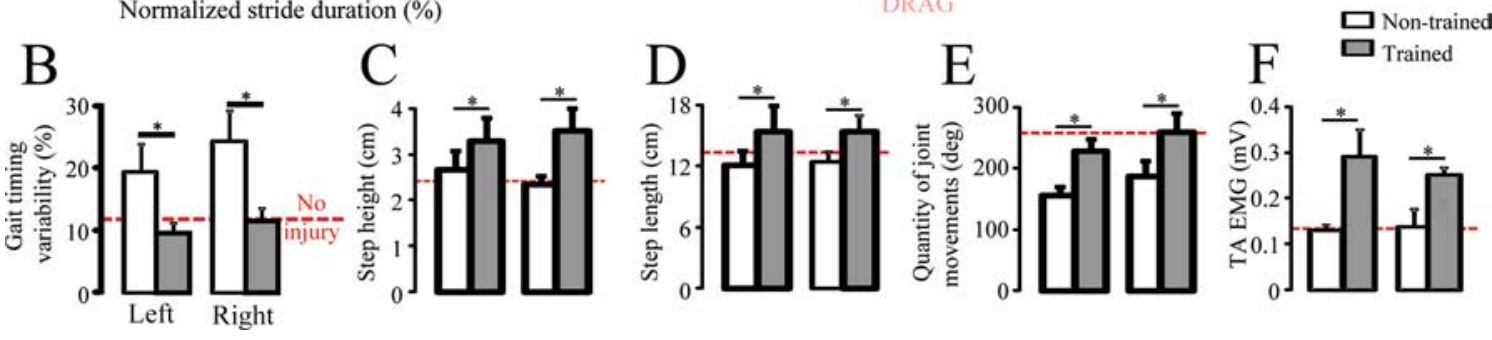

Figure 1. A, Stick diagram decomposition, joint angular displacement, and EMG activity of selected hindlimb muscles of a step cycle during testing in a representative noninjured, spinal rat without ES or quipazine (Q), and nontrained and step-trained rat with $\mathrm{ES}(40 \mathrm{~Hz}$ at L2) and Q $(0.3 \mathrm{mg} / \mathrm{kg}) . \boldsymbol{B}$, Gait timing variability, an indicator of interim coordination variability, was higher in nontrained than noninjured or trained rats. Step height $(\boldsymbol{C})$ and length $(\boldsymbol{D})$, total quantity of joint movement $(\boldsymbol{E})$, and TA EMG amplitude $(\boldsymbol{F})$ were greater in trained than nontrained rats. The horizontal red dotted line in $\boldsymbol{B}-\boldsymbol{F}$ indicates values for noninjured rats. Error bars indicate SEM. ${ }^{*} p<0.05$, significant difference between trained and nontrained groups. MTP, Metatarsophalangeal joint; VL, vastus lateralis; St, semitendinosus; MG, medial gastrocnemius; TA, tibialis anterior.

number of FOS + nuclei between trained and nontrained rats in laminas I, VIII, IX, Clarke's column, and intermediolateral column (IML) (Fig. 3D). In contrast, there were more FOS + nuclei in nontrained than trained rats in laminas II, III, IV, V, VI, VII $(p<0.001)$, and $\mathrm{X}(p<0.05)$.

\section{Discussion}

A novel locomotor pattern emerged when complete spinal adult rats were trained to step bipedally under ES and quipazine

The locomotor properties in the trained spinal rats were different from both the noninjured and nontrained spinal rats. Unlike nontrained rats, trained rats were able to produce consistent stepping. Those steps had greater amplitude (length and height) and a narrower base of support than both the noninjured and nontrained rats. Repeated exposure to the stepping task resulted in well coordinated stepping that could sustain the injured rat in a stable dynamic posture during locomotion. Nontrained rats adopted a wider stance posture, similar to noninjured animals. It was apparent, however, that such posture was ineffective in keeping these injured animals stable. They demonstrated more dragging of the toes during the initial stages of the swing phase, and greater gait timing variability. Trained rats also demonstrated increased facilitation of the ankle flexors, which could have been an effect of the repeated administration of quipazine during the training. In addition to changes in the sensorimotor pathways (Côté and Gossard, 2004), locomotor training has been shown to increase segmental and central EPSPs and decrease afterhyperpolarization depth, which lead to facilitation of motoneuron activation (Petruska et al., 2007).

We showed previously that the level of ES and quipazine used in the present study does not directly induce stepping, but enables the spinal cord to respond appropriately to the proprioceptive input associated with weight bearing on a moving treadmill belt (Gerasimenko et al., 2007). Here, we demonstrate that repetitive patterns of this afferent information associated with weightbearing stepping allow the spinal cord to find stable solutions to a complex motor problem (i.e., stepping on a moving treadmill belt). A similar phenomenon has been described in adult cats in which daily locomotor training resulted in longer sustained bouts of stepping (Lovely et al., 1990; De Leon et al., 1999). In the present study, trained rats were not only able to step continuously for longer periods and at faster speeds (data not shown), but most importantly they demonstrated a unique gait pattern.

The levels of ES and the dosages of quipazine administered for 


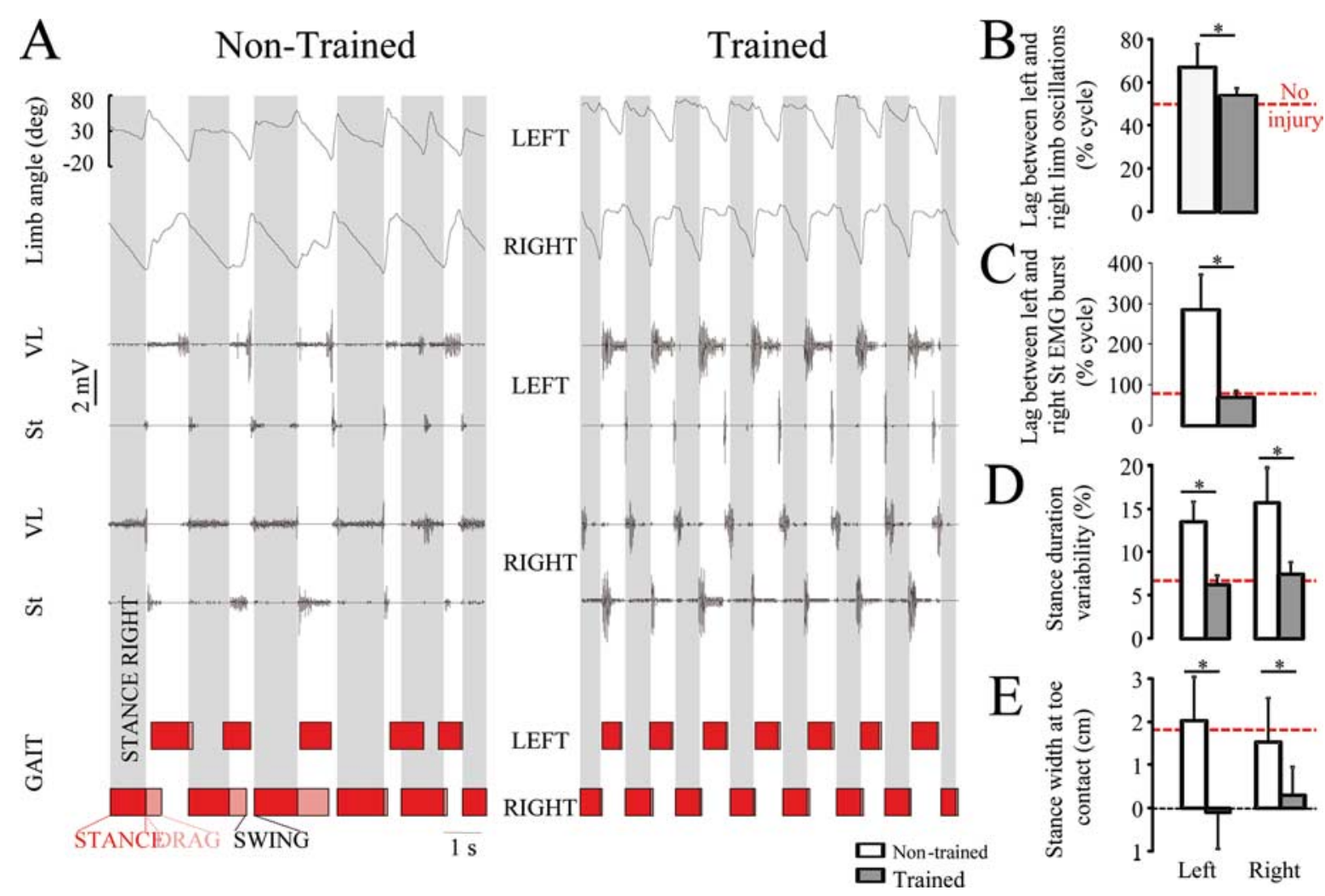

Figure 2. A, Representative EMG recordings from the left and right VL and St, with corresponding gait cycle diagrams for a nontrained and a trained rat treated with ES and Q. B, C, The left and right limbs moved out of phase in both noninjured and trained rats $(\boldsymbol{B})$, whereas nontrained rats showed impaired interlimb coordination and a significant lag between the left and right $S t$ EMG bursts over the duration of the stepping sequence $(\boldsymbol{C}) \cdot \boldsymbol{D}, \boldsymbol{E}$, Variability of stance duration (computed as coefficient of variability) $(\boldsymbol{D})$ and stance width at toe contact $(\boldsymbol{E})$ were greater in nontrained than trained rats. The horizontal red dotted line in $\boldsymbol{B}-\boldsymbol{E}$ indicates control values for noninjured rats. Error bars indicate $S E M .{ }^{*} p<0.01$, significant difference between trained and nontrained groups. Abbreviations are the same as in Figure 1.

42 consecutive days were constant and sufficient to facilitate successful locomotor behaviors. Spinal rats were not able to produce reliable locomotion without ES and/or quipazine treatment, not even the trained group (Fig. $1 A$ ). A quipazine treatment-only control group was not added because we showed previously that repeated quipazine administration alone does not affect locomotor ability (Fong et al., 2005).

\section{Spinal circuits associated with stepping become more specific,} localized, and efficient with step training

The general pattern and activation levels of spinal neurons after a single bout $(1 \mathrm{~h})$ of stepping in the nontrained and step-trained spinal rats are similar to those observed previously in nontrained noninjured rats (Ahn et al., 2006) and in decerebrated cats during brainstem stimulation (Dai et al., 2005). It is obvious from the marked lower number of FOS + nuclei in the trained than nontrained rats that aberrant spinal circuits were activated in the nontrained rats. This reorganization has been shown neurochemically, structurally, and electrophysiologically, as well as behaviorally after a complete spinal cord transection (Tillakaratne et al., 2000, 2002; Petruska et al., 2007). The spinal circuitry that generates stepping movements also spontaneously reorganizes after incomplete spinal cord injuries (Bareyre et al., 2004; Courtine et al., 2008). These changes would seem to include the emergence of aberrant functional connections and could contribute to the poorly coordinated pattern of motor pool activation typically seen after a complete spinal cord transection. This interpretation is consistent with the larger number of FOS + neurons observed in the nontrained compared with trained spinal rats.
Daily locomotor training of chronic spinal animals tends to normalize many properties of the spinal cord after an injury (for review, see Edgerton et al., 2008). Functionally, it appears that the pathways that are more efficient in performing the task successfully can be activated with greater consistency after a training period. The efficacy of specific connections is likely to be strengthened with practice, as observed after learning in the hippocampus and functional magnetic resonance imaging studies in humans learning movement sequences. In the spinal cord, Petruska et al. (2007) demonstrated that, in rats spinalized as neonates (postnatal day 5), locomotor training resulted in increased motoneuron EPSPs, and decreases in afterhyperpolarization depth when compared with nontrained spinal rats, suggesting specific electrophysiological responses to training. A significant decrease in FOS IHC after exercise training also has been reported for specific brain nuclei related to the control of cardiorespiratory function (Ichiyama et al., 2002). An increase in efficacy is further illustrated by the fact that, although trained spinal rats had increased TA EMG activation during stepping, apparently fewer spinal neurons driving the muscle contractions were activated compared with nontrained rats. However, although we report significant changes in FOS IHC, other anatomical changes (e.g., dendritic and synaptic plasticity, neurite sprouting) may also be occurring. Nonetheless, these results raise the issue of which specific interneuronal populations may be responsible for the renewed capability to coordinate the motoneuronal pools more effectively after step training. 
A T12

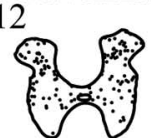

T13

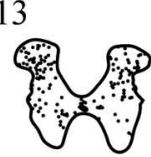

L1

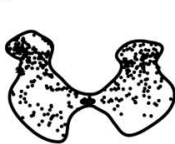

L2
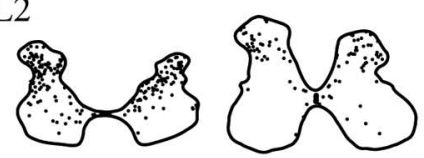

L3

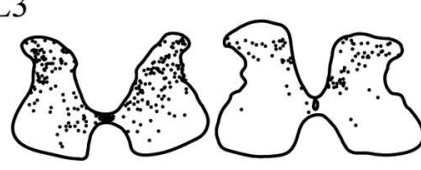

L4
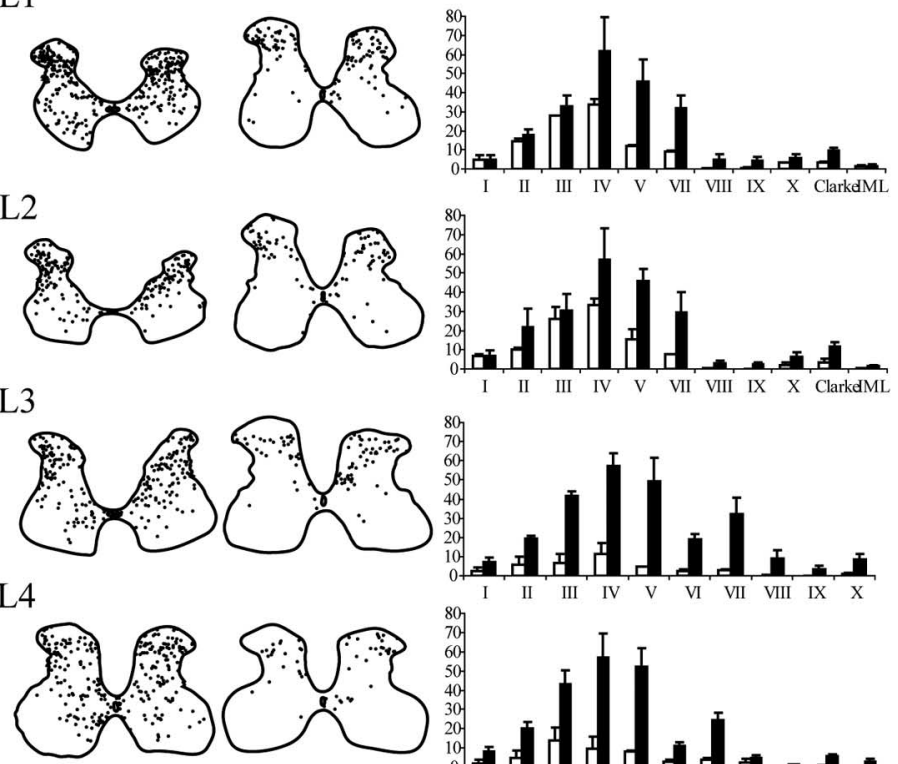

L5
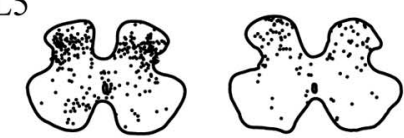

L6

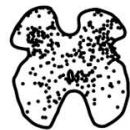

S1

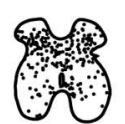

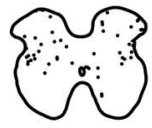

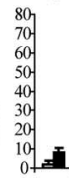

80
70
60
50
40
30
20
10
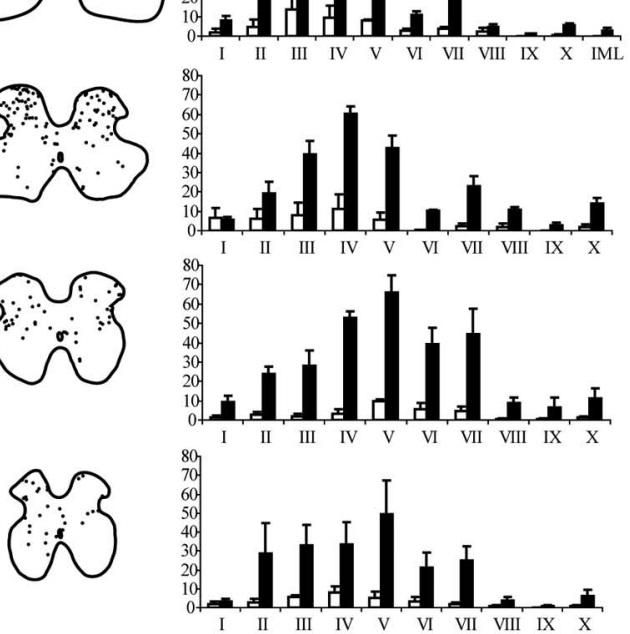

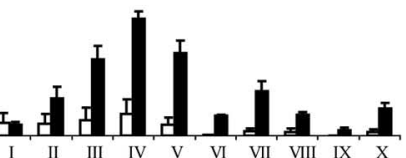

B

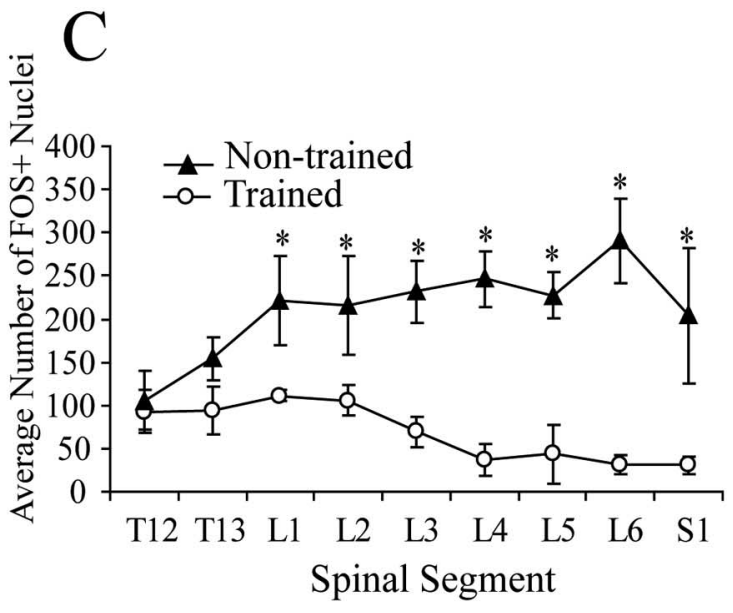

D

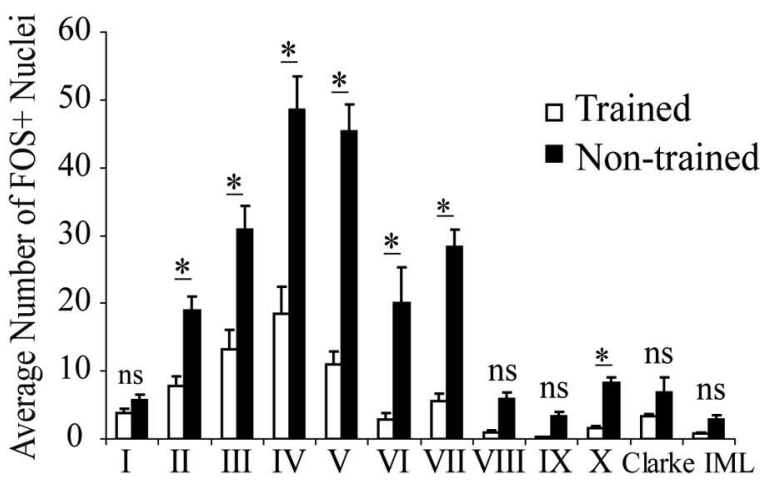

Spinal Laminae

E

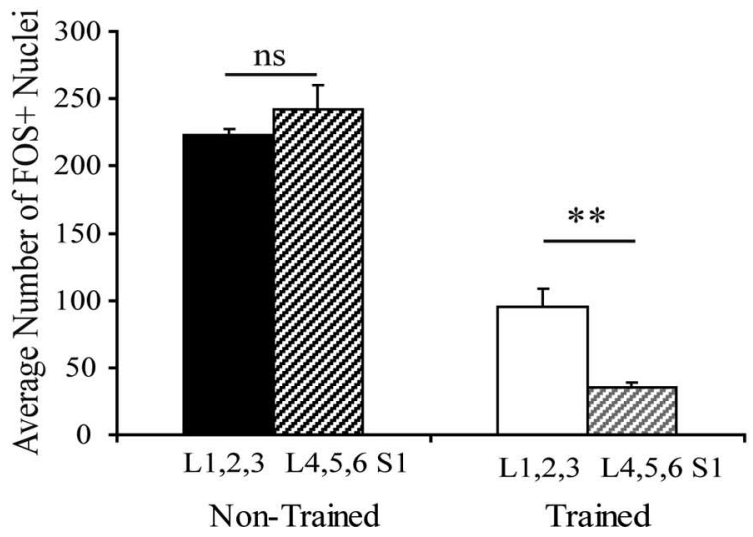

Figure 3. FOS immunohistochemistry after $1 \mathrm{~h}$ bipedal stepping under ES $(\mathrm{L2} ; 40 \mathrm{~Hz}) . \mathbf{A}$, Camera lucida drawing of the location of FOS + nuclei for a representative nontrained and trained spinal rat. Note that FOS + nuclei were distributed in all segments, and all laminas were analyzed in both groups. $\boldsymbol{B}$, Mean ( \pm SEM) number of FOS + for each lamina at each spinal segment is shown. $\boldsymbol{C}$, The average number of FOS + nuclei per spinal segment was greater in nontrained than trained rats in spinal segments L1 to S1. D, Differences in the mean ( \pm SEM) number of FOS + nuclei per lamina for all spinal segments combined between spinal trained and nontrained rats are shown. ${ }^{*} p<0.05$, significant difference between trained and nontrained groups. $\boldsymbol{E}$, Comparison of the average number of FOS + nuclei in the rostral $(\mathrm{L} 1, \mathrm{~L} 2, \mathrm{~L} 3)$ and caudal $(\mathrm{L} 4, \mathrm{~L} 5, \mathrm{~L} 6 ; \mathrm{S} 1)$ lumbosacral segments. Trained rats had significantly $(p<0.01)$ fewer F0S + nuclei in the caudal segments, whereas nontrained rats did not show such a difference. ns, Nonsignificant.

Which spinal circuits are necessary for locomotion?

The observation that only lumbosacral spinal segments were significantly affected by training suggests that circuits specifically driving this task must be located in those segments, which is in agreement with a large body of evidence (Kiehn, 2006). In the trained spinal rats, the rostral lumbar segments (L1-L3) had sig- nificantly more FOS + nuclei than the caudal segments $(p<$ $0.005)$ (Fig. 3E). This difference was not observed in nontrained rats. Most motor pools of the hindlimb muscles are concentrated in the caudal lumbar segments (Vanderhorst and Holstege, 1997). This observation is also in agreement with the notion that the rhythmogenic centers in the spinal cord reside within the 
rostral lumbar segments (Grillner and Zangger, 1979; Kjaerulff and Kiehn, 1996; Bonnot and Morin, 1998; Kiehn and Kjaerulff, 1998). These observations were masked in nontrained rats perhaps because of the redundant activation of neurons. The significantly lower number of FOS + nuclei in laminas II, III, IV, V, VI, VII, and X in trained than nontrained rats strongly suggests that the redundant connections were mostly related to computation of afferent input by neurons in those laminas. Even after training, however, FOS + nuclei were observed in every lamina, suggesting that a subset of those neurons are necessary for locomotion, and that the circuitry for stepping is distributed throughout all laminas along the extent of the lumbosacral spinal cord. Because it has been reported that not all active spinal neurons show FOS + IHC (Dragunow and Faull, 1989; Carr et al., 1995), the absence of detectable FOS should be interpreted with caution. Neither can it be discounted that the training itself may have reduced the responsiveness of the c-fos activation to activity. Finally, it is also plausible, albeit unlikely, that the significant reduction in FOS+ neurons in the trained rats could be attributable, in part, to daily administration of quipazine, ES, or some combination of the two.

\section{Summary}

We demonstrated that, given enough physiological activation, afferent input can drive spinal circuits to enable stepping and that repetitive practice allows the isolated lumbosacral circuits to find new solutions in executing motor and postural problems imposed by a spinal cord injury. The observations that stepping is more consistent and that fewer neurons are activated in trained than nontrained spinal rats during stepping suggest a selective reinforcement of specific sensorimotor pathways within the spinal cord with motor training.

\section{References}

Ahn SN, Guu JJ, Tobin AJ, Edgerton VR, Tillakaratne NJ (2006) Use of c-fos to identify activity-dependent spinal neurons after stepping in intact adult rats. Spinal Cord 44:547-559.

Bareyre FM, Kerschensteiner M, Raineteau O, Mettenleiter TC, Weinmann O, Schwab ME (2004) The injured spinal cord spontaneously forms a new intraspinal circuit in adult rats. Nat Neurosci 7:269-277.

Bonnot A, Morin D (1998) Hemisegmental localisation of rhythmic networks in the lumbosacral spinal cord of neonate mouse. Brain Res 793:136-148.

Carr PA, Huang A, Noga BR, Jordan LM (1995) Cytochemical characteristics of cat spinal neurons activated during fictive locomotion. Brain Res Bull 37:213-218.

Côté MP, Gossard JP (2004) Step training-dependent plasticity in spinal cutaneous pathways. J Neurosci 24:11317-11327.

Courtine G, Roy RR, Raven J, Hodgson J, McKay H, Yang H, Zhong H, Tuszynski MH, Edgerton VR (2005) Performance of locomotion and foot grasping following a unilateral thoracic corticospinal tract lesion in monkeys (Macaca mulatta). Brain 128:2338-2358.

Courtine G, Song B, Roy RR, Zhong H, Herrmann JE, Ao Y, Qi J, Edgerton VR, Sofroniew MV (2008) Recovery of supraspinal control of stepping via indirect propriospinal relay connections after spinal cord injury. Nat Med 14:69-74.

Dai X, Noga BR, Douglas JR, Jordan LM (2005) Localization of spinal neurons activated during locomotion using the $\mathrm{c}$-fos immunohistochemical method. J Neurophysiol 93:3442-3452.

De Leon RD, Hodgson JA, Roy RR, Edgerton VR (1999) Retention of hind- limb stepping ability in adult spinal cats after the cessation of step training. J Neurophysiol 81:85-94.

Dragunow M, Faull R (1989) The use of c-fos as a metabolic marker in neuronal pathway tracing. J Neurosci Methods 29:261-265.

Edgerton VR, Kim SJ, Ichiyama RM, Gerasimenko YP, Roy RR (2006) Rehabilitative therapies after spinal cord injury. J Neurotrauma 23:560-570.

Edgerton VR, Courtine G, Gerasimenko YP, Lavrov I, Ichiyama RM, Fong AJ, Cai LL, Otoshi CK, Tillakaratne NJ, Burdick JW, Roy RR (2008) Training locomotor networks. Brain Res Rev 57:241-254.

Fong AJ, Cai LL, Otoshi CK, Reinkensmeyer DJ, Burdick JW, Roy RR, Edgerton VR (2005) Spinal cord-transected mice learn to step in response to quipazine treatment and robotic training. J Neurosci 25:11738-11747.

Gerasimenko YP, Ichiyama RM, Lavrov IA, Courtine G, Cai L, Zhong H, Roy RR, Edgerton VR (2007) Epidural spinal cord stimulation plus quipazine administration enable stepping in complete spinal adult rats. J Neurophysiol 98:2525-2536.

Grillner S, Zangger P (1979) On the central generation of locomotion in the low spinal cat. Exp Brain Res 34:241-261.

Hodgson JA, Roy RR, Higuchi N, Monti RJ, Zhong H, Grossman E, Edgerton VR (2005) Does daily activity level determine muscle phenotype? J Exp Biol 208:3761-3770.

Ichiyama RM, Gilbert AB, Waldrop TG, Iwamoto GA (2002) Changes in the exercise activation of diencephalic and brainstem cardiorespiratory areas after training. Brain Res 947:225-233.

Ichiyama RM, Gerasimenko YP, Zhong H, Roy RR, Edgerton VR (2005) Hindlimb stepping movements in complete spinal rats induced by epidural spinal cord stimulation. Neurosci Lett 383:339-344.

Ichiyama RM, Gerasimenko Y, Jindrich DL, Zhong H, Roy RR, Edgerton VR (2008) Dose dependence of the 5-HT agonist quipazine in facilitating spinal stepping in the rat with epidural stimulation. Neurosci Lett 438:281-285

Kiehn O (2006) Locomotor circuits in the mammalian spinal cord. Annu Rev Neurosci 29:279-306.

Kiehn O, Kjaerulff O (1998) Distribution of central pattern generators for rhythmic motor outputs in the spinal cord of limbed vertebrates. Ann N Y Acad Sci 860:110-129.

Kjaerulff O, Kiehn O (1996) Distribution of networks generating and coordinating locomotor activity in the neonatal rat spinal cord in vitro: a lesion study. J Neurosci 16:5777-5794.

Lovely RG, Gregor RJ, Roy RR, Edgerton VR (1990) Weight-bearing hindlimb stepping in treadmill-exercised adult spinal cats. Brain Res 514:206-218.

Molander C, Xu Q, Grant G (1984) The cytoarchitectonic organization of the spinal cord in the rat. I. The lower thoracic and lumbosacral cord. J Comp Neurol 230:133-141.

Petruska JC, Ichiyama RM, Jindrich DL, Crown ED, Tansey KE, Roy RR, Edgerton VR, Mendell LM (2007) Changes in motoneuron properties and synaptic inputs related to step training after spinal cord transection in rats. J Neurosci 27:4460-4471.

Tillakaratne NJ, Mouria M, Ziv NB, Roy RR, Edgerton VR, Tobin AJ (2000) Increased expression of glutamate decarboxylase $(\mathrm{GAD}(67))$ in feline lumbar spinal cord after complete thoracic spinal cord transection. J Neurosci Res 60:219-230.

Tillakaratne NJ, de Leon RD, Hoang TX, Roy RR, Edgerton VR, Tobin AJ (2002) Use-dependent modulation of inhibitory capacity in the feline lumbar spinal cord. J Neurosci 22:3130-3143.

Timoszyk WK, Nessler JA, Acosta C, Roy RR, Edgerton VR, Reinkensmeyer DJ, de Leon R (2005) Hindlimb loading determines stepping quantity and quality following spinal cord transection. Brain Res 1050:180-189.

Vanderhorst VG, Holstege G (1997) Organization of lumbosacral motoneuronal cell groups innervating hindlimb, pelvic floor, and axial muscles in the cat. J Comp Neurol 382:46-76. 